\title{
Electron gun for diffraction experiments on controlled molecules
}

\author{
Nele L. M. Müller, ${ }^{1}$ Sebastian Trippel, ${ }^{1}$ Karol Długołęcki, ${ }^{1}$ and Jochen Küpper ${ }^{1,2,3, 冈 ~}$ \\ ${ }^{1}$ Center for Free-Electron Laser Science, DESY, Notkestrasse 85, 22607 Hamburg, Germany \\ ${ }^{2}$ The Hamburg Center for Ultrafast Imaging, University of Hamburg, Luruper Chaussee 149, 22761 Hamburg, Germany \\ ${ }^{3}$ Department of Physics, University of Hamburg, Luruper Chaussee 149, 22761 Hamburg, Germany
}

(Dated: July 10, 2015)

\begin{abstract}
A dc electron gun, generating picosecond pulses with up to $8 \times 10^{6}$ electrons per pulse, was developed. Its applicability for future time-resolved-diffraction experiments on state- and conformer-selected laser-aligned or oriented gaseous samples was characterized. The focusing electrodes were arranged in a velocity-map imaging spectrometer configuration. This allowed to directly measure the spatial and velocity distributions of the electron pulses emitted from the cathode. In combination with electron trajectory simulations, this permitted the characterization of the electron beam in terms of coherence length and pulse duration. Electron diffraction data of a thin aluminum foil illustrated the diffraction capabilities of the electron-gun setup.
\end{abstract}

Diffraction methods have proven to be a powerful tool for investigating the structures of solid state samples [1], for example in transmission electron microscopy [2] or $x-$ ray crystallography [3, 4]. Electron diffraction has found broad application for gas-phase structure-determination in chemistry [5]. Recent developments have mainly focused on realizing time-resolved experiments in order to study structural changes, where x-ray and electron diffraction served as complementary approaches [6-11].

The first sources for creating electron pulses short enough to study ultrafast processes in molecules or materials were dc electron guns. Here, electrons were created from metallic surfaces by short laser pulses and accelerated in dc electric fields [7, 8], yielding sub-picosecond electron pulses of moderate coherence and brilliance. Radio-frequency cavities allow for temporal compression of electron pulses through phase-space rotation [12], shortening the pulse duration to below $100 \mathrm{fs}$ with electron numbers of $10^{6}$ per pulse and electron spot sizes below $100 \mu \mathrm{m}$. Compact dc guns can achieve comparable properties by increasing the acceleration fields and reducing the path length, during which the electron pulse can expand [13-15]. Ultra-fast-single-electron sources [16] avoid the problem of space charges, but rely on very high repetition rates to achieve sufficient electron fluxes for diffractive imaging experiments. The use of ultra cold atoms as electron sources [17, 18] increases the coherence. Other possible sources for time-resolved electron diffraction are low energy electron setups [19] or laser-induced electron diffraction [20, 21].

For studies of gas-phase molecules the information that can be retrieved from a diffraction pattern can be enhanced by controlling the spatial orientation of the molecules. This was proposed theoretically [22-25] and demonstrated experimentally for x-ray [26, 27] as well as for electron diffraction [28, 29]. While laser-alignment of molecules allows for three-dimensional reconstruction of the molecular structure [29], generally strong alignment or orientation is necessary [11, 25]. In order to achieve high degrees of alignment and orientation and, hence, to allow for a better reconstruction of threedimensional structures, the molecules can be cooled in a supersonic expansion and subsequently rotational-state selected through electric fields [30-32].

If the molecular samples are prepared in this controlled way, their densities are on the order of $10^{8}$ molecules $/ \mathrm{cm}^{3}$ [26, 33]. The calculated cross section for a prototypical molecule, containing two iodine atoms, is on the order of $10^{-15} \mathrm{~cm}^{2}$ for a solid angle of $1.3 \times 10^{-3}$ sr blocked by a beam stop. For laser alignment the molecules are typically exposed to laser fields with intensities of $1 \mathrm{TW} / \mathrm{cm}^{2}$, which can be achieved by tightly focusing the laser beam [34]. This results in an interaction volume on the order of $1 \times 10^{-6} \mathrm{~cm}^{3}$. The small number of molecules present in this volume implies the need for bright electron sources with $10^{9}$ electrons per second. For experimental repetition rates on the order of $1 \mathrm{kHz}$ [35], this corresponds to $10^{6}$ electrons per shot with a electron beam focus size of approximately $100 \mu \mathrm{m}$. The setup presented here produced the necessary electron numbers and allowed for a characterization of the electron beam to ensure, for instance, the required transverse coherence length.

A schematic of the experimental setup is shown in Figure 1 The electrons were photo-emitted from a copper cathode via one-photon absorption after irradiation with short UV laser pulses. The pulses were generated by third-harmonic generation (THG) of 30-fs-duration nearinfrared pulses from a Ti:Sapphire laser (TSL) system with a repetition rate of $1 \mathrm{kHz}$. Based on the pulse duration of the near-infrared laser pulse at the THG setup, and the dispersion in subsequent optical elements, we estimated a pulse duration of $370 \mathrm{fs}$ for the UV light. The central wavelength was $265 \mathrm{~nm}$ with a spectral width of $4 \mathrm{~nm}$. The pulse impinged on the cathode under an angle of $20^{\circ}$ to the cathode surface. The electrons were accelerated and focused by three electrodes in velocity-map imaging spectrometer (VMI) configuration. The applied potential at the cathode was $U_{c}=-15 \mathrm{kV}$. The voltage on the focusing electrode was varied between $U_{f}=0 \mathrm{kV}$ and 


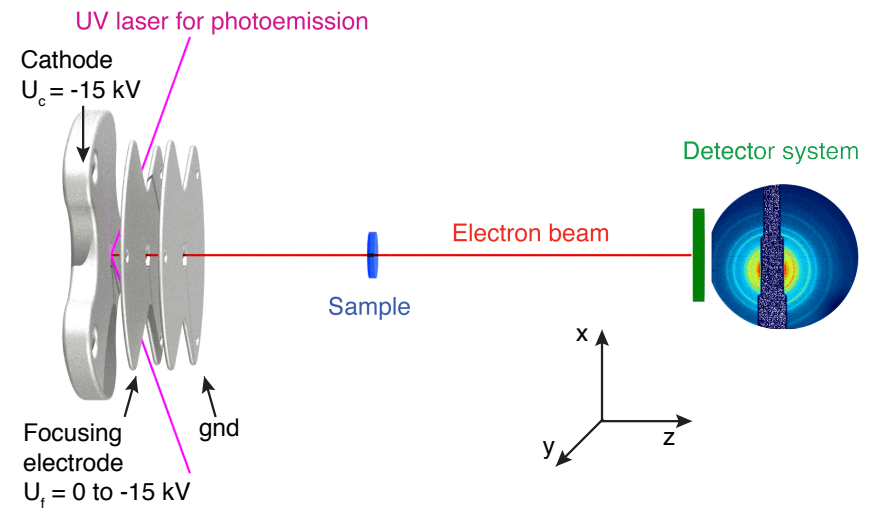

FIG. 1. Experimental scheme of the electron gun consisting of three electrodes in velocity-map-imaging configuration. After photoemission, the electrons were diffracted of an aluminum sample or were measured by a Faraday cup coupled to an electrometer. A multi-channel-plate detector with phosphor screen and a camera was used for position sensitive detection.

$-15 \mathrm{kV}$. The third electrode was kept on ground potential. The asymmetric electrode shape allowed the laser beam to pass and impinge on the cathode. The holes within electrodes were large enough to avoid clipping of the electron beam. On the one hand this reduced the background signal in diffraction experiments, as there was no electron scattering off the electrodes. On the other hand it allowed for steering the electron beam's position by changing the laser-spot position on the cathode. The voltage on the focusing electrode $U_{f}$ determined the position of the electron beam focus along the $z$ direction. The electrode configuration allowed to characterize the electron pulse by applying the corresponding voltages for spatialand velocity-mapping [36, 37]. The spatial distribution of the electron beam was recorded by a position sensitive detector consisting of a multi-channel-plate (MCP) with a phosphor screen and a CMOS camera (Optronis CamRecord CL600x2). The detection system was read out with a $1 \mathrm{kHz}$ repetition rate, which allowed for single electron counting in the case of a few electrons per pulse. At high electron numbers, the gain of the detector had to be reduced to avoid damage of the MCP. With lower gain single electrons could not be resolved anymore. A Faraday cup connected to an electrometer (Keithley 6514 electrometer) was used to measure the electron number per pulse. To further characterize the electron pulses, we performed diffraction experiment with a thin aluminum foil on a TEM grid, which was introduced into the electron beam path. In this case the direct electron beam was blocked by a copper or aluminum beam block.

The electron gun was designed for ultra-high vacuum. Here, the final pressure was $4 \times 10^{-9} \mathrm{mbar}$ using a turbomolecular pump with pumping speed of $300 \mathrm{l} / \mathrm{s}$, limited by outgassing from the cable of the Faraday cup and from PEEK material of the electron gun insulators. This low

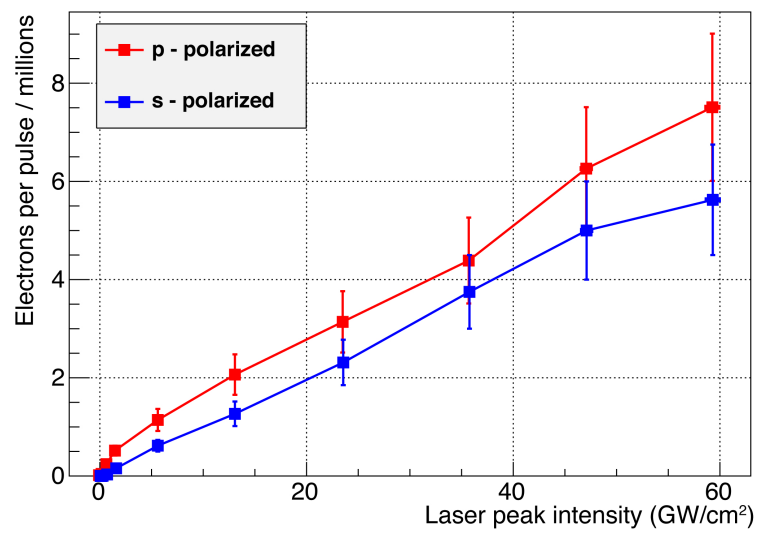

FIG. 2. Electron number in dependence of laser peak intensity for p-polarized (red) and s-polarized (blue) laser light and a central laser wavelength of $265 \mathrm{~nm}$.

pressure is essential when investigating thin gas-phase samples in order to reduce the scattering by background gas. We expect to achieve pressures of some $10^{-10}$ in the final setup when replacing all PEEK insulators by MACOR or alumina.

In Figure 2 the electron number per pulse is shown for $U_{f}=-13.2 \mathrm{kV}$ in dependence of the laser pulse intensity for two laser polarizations. This corresponded to the focus of the electron beam being close to the detector surface. The number of electrons increased linearly with the laser power, as expected for a one-photon effect of $265 \mathrm{~nm}$ light with a spectral width of $4 \mathrm{~nm}$ on copper, which has a work function of $4.7 \mathrm{eV}$. No saturation was observed. The number of generated electrons depended on the laser polarization: For p-polarization (red curve, field vector in plane of incidence) more electrons were generated than for s-polarization (blue curve, field vector parallel to cathode surface). This is in accord with the reflectivity of copper being higher for s-polarized light than for p-polarized light, which was confirmed by measuring the laser power for both polarizations after the cathode. Up to $8 \times 10^{6}$ electrons per shot could be obtained, which is sufficient for the planned diffraction experiments on dilute gas-phase samples delivered by the controlled-molecules apparatus.

For a full characterization of the electron beam the electron spot size at the detector was measured for various focusing voltages $U_{f}$, including those for spatial- and velocity-mapping. In Figure 3 a the root-mean-square (RMS) spot size of the electron beam in $x$ and $y$ dimension, $\sigma_{x}$ and $\sigma_{y}$, are plotted as a function of $U_{f}$. Here, approximately five electrons per pulse were emitted from the cathode and, therefore, space charge effects were negligible. The spot size decreased with increasing $U_{f}$ until it reached a focus, at the detector, for about $U_{f}=-13 \mathrm{kV}$. Raising $U_{f}$ further led to a defocusing of the electron 
a

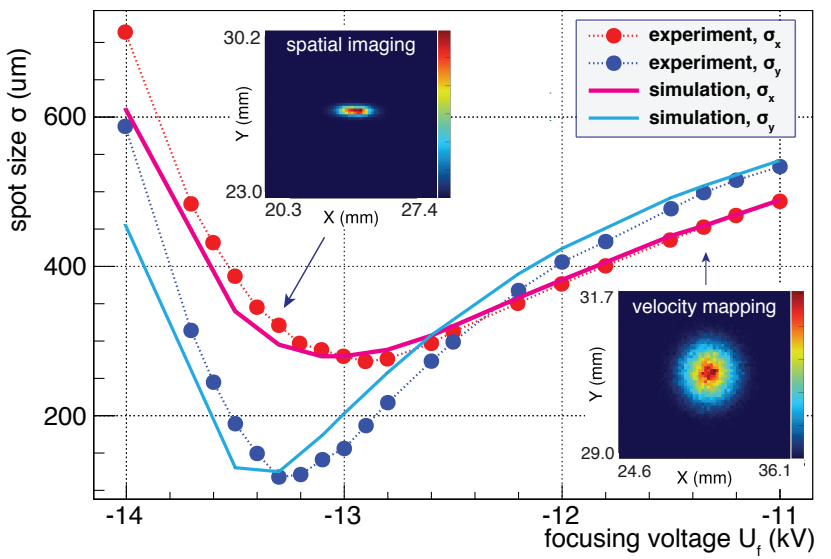

b

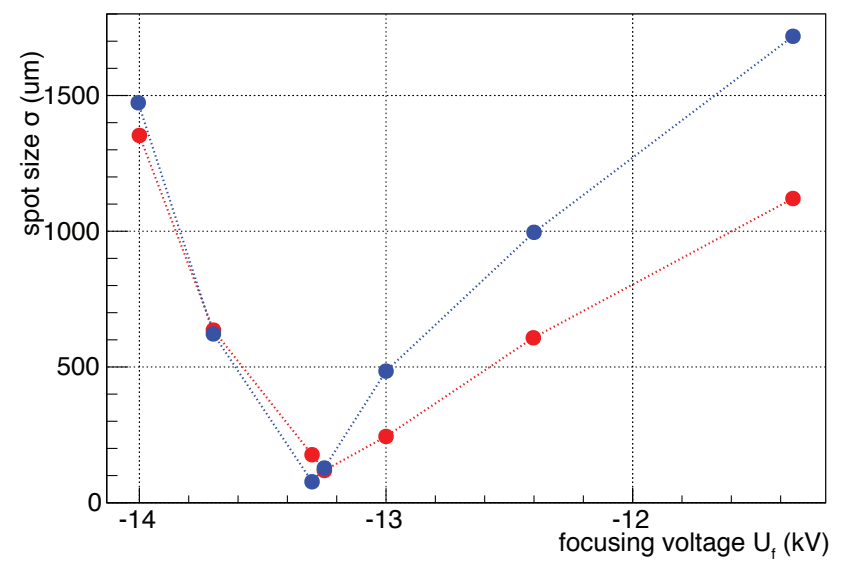

FIG. 3. Electron spot size on the detector for different focusing voltages for (a) a few and (b) $2 \times 10^{5}$ electrons per pulse. Red and blue dots are experimental results corresponding to $x$ and $y$ directions, respectively. Magenta and cyan lines correspond to corresponding simulations in $x$ and $y$ dimension. The insets in (a) show detector images for spatial (left) and velocity map imaging (right).

beam, i.e., the focus was placed before the detector. The exact voltage to place the focus onto the detector depends on the initial size of the electron cloud. The electron beam was broadened in $x$ direction due to the small angle of incidence of the laser. Therefore, the foci in $x$ and $y$ dimension had slightly different focusing behavior.

In spatial imaging mode, $U_{f}=-13.3 \mathrm{kV}$, the spatial distribution of emitted electrons was mapped onto the detector, which is depicted as inset in Figure 3a. In this case, all electrons, which started from a certain coordinate on the cathode hit a corresponding point on the detector, in first order independent of their momentum [36]. The magnification factor $m_{s}$ for spatial imaging was calibrated in the experiment by translating the laser-focus spot on the cathode using the focusing lens. From a known displacement of the electron beam on the cathode $\Delta x_{C}$ and the corresponding measured displacement of the electrons on the detector $\Delta x_{D}$ a magnification factor of $m_{s}=\Delta x_{D} / \Delta x_{C}=3.9$ was determined. This agreed with the simulated value. For simulations of electric fields and trajectories finite-element methods were used (COMSOL Multiphysics). The inferred RMS sizes of the electron beam at the cathode were $\sigma_{x}=85(3) \mu \mathrm{m}$ and $\sigma_{y}=31(1) \mu \mathrm{m}$; values in parenthesis depict one standard deviation. The difference in spread in the two dimensions agreed well with the effective broadening of the photoemission laser by a factor of approximately three in $x$ direction due to its angle of incidence of $20^{\circ}$.

In velocity map imaging mode $\left(U_{f}=-11.35 \mathrm{kV}\right)$ the transverse velocity distribution was mapped onto the detector, which is shown as the second inset in Figure 3 a. The velocity spread was similar in both dimensions. With the simulated magnification factor of $m_{v}=0.9$ for velocity mapping and a simulated electron time of flight of $4.1 \mathrm{~ns}$ an energy spread of $\sigma_{E}=0.1 \mathrm{eV}$ was obtained. This agrees well with $\sigma_{E}=0.13 \mathrm{eV}$ reported in [38].

In order to characterize the electron beam further simulations and measurements at various focusing voltages $U_{f}$ were performed. The spatial and velocity distribution of the electrons in $x$ and $y$ dimension could be retrieved from the experiment, but the corresponding values in $z$ dimension had to be simulated. Electric fields were calculated using finite-element methods (COMSOL Multiphysics) and the electron trajectories in these fields were simulated using ASTRA [39]. The initial spatial distribution at the cathode was taken from the measurements described above. Together with a Fermi Dirac distribution for the one-photo-emission [39], this led to the emittance values of $\epsilon_{x}=0.026 \pi \mathrm{mrad} \mathrm{mm}$ and $\epsilon_{y}=0.010 \pi \mathrm{mrad} \mathrm{mm}$, and the energy spread in $z$-direction of $\sigma_{E_{z}}=0.2 \mathrm{eV}$. Fitting the emittance to the transverse velocity distributions retrieved from VMI mode, while keeping $\sigma_{E_{z}}$ constant, resulted in $\epsilon_{x}=0.029 \pi \mathrm{mrad} \mathrm{mm}$ and $\epsilon_{y}=0.012 \pi \mathrm{mrad} \mathrm{mm}$, in good agreement with the values obtained from the Fermi Dirac distribution. Using the fitted input parameters, the overall dependence of the electron beam spot size at the detector on the focusing voltage was simulated. The results are depicted by the magenta and cyan lines in Figure 3 a and are, again, in good agreement with the experimental results. This indicates that also the simulated $\sigma_{E_{z}}$ was sensible. Due to the good agreement between experiment and simulation it is possible to deduce properties of the electron beam from the simulations, including size, coherence length and pulse duration, for its whole propagation. The coherence length at the sample position $(11 \mathrm{~cm}$ downstream from the cathode) was deduced to be $3 \mathrm{~nm}$ in $\mathrm{x}$-dimension and $1.2 \mathrm{~nm}$ in $\mathrm{y}$-dimension. The pulse duration at this position was $1.4 \mathrm{ps}$.

Figure 3 b shows the spot sizes for $2 \times 10^{5}$ electrons per pulse, where space charges had a significant effect. For the detection of $2 \times 10^{5}$ electrons per pulse in Figure $3 \mathrm{~b}$, the detector voltage had to be reduced and single electron detection was not possible. This implies that 
Figure $3 \mathrm{a}$ and $\mathrm{b}$ are only qualitatively comparable. A stronger asymmetry in velocity map imaging mode was observed than above. This could not be reproduced using a cylindrical symmetry in electric fields and initial velocities, which was a good approximation in the simulations for few electrons. Using finite-element simulations (COMSOL Multiphysics) it was possible to qualitatively determine the origin of the asymmetry in the velocity map imaging mode, but a full simulation of all 3D trajectories for $2 \times 10^{5}$ electrons was not possible due to too high computational cost. Simulations for few electrons (COMSOL Multiphysics) showed that the trajectories of the electrons far off the central axis of the spectrometer were disturbed by the asymmetry of the electric field due to the opening in the electrodes, see Figure 1 . This became more pronounced when space charges lead to a significant broadening of the electron distribution. In the case of $2 \times 10^{5}$ electrons per pulse the radial distribution between the cathode and the focusing electrode was increased by an order of magnitude compared to the few electron case. This lead to a larger magnification factor in vertical direction in velocity map imaging mode and, therefore, contributed to the asymmetry in the detector image. Secondly, the space charge effect itself lead to an asymmetry in the velocity distribution, if the electron spot was asymmetric. Assuming a cylindrical shape of an electron cloud with homogeneous density, it can be shown, that the acceleration force due to space charge in the direction of the smaller dimension is stronger [40]. Moreover, simulating (COMSOL Multiphysics) similar electron densities in smaller, but asymmetric volumes showed an asymmetric velocity distribution. Both effects resulted in the vertical broadening of the electron pulse in velocity-mapping mode.

Simulations in cylindrical symmetry (ASTRA) for one million electrons per pulse provided an approximate value for the pulse duration at the sample position of $60 \mathrm{ps}$. Albeit this was much longer than in the case of a few electrons/pulse, it is sufficiently short for the diffractive imaging of aligned and oriented molecules, which we can routinely create and control for hundreds of picoseconds [31, 35].

A thin polycrystalline aluminum sample was used to test the electron-optical properties of the generated electron pulses, for instance, its coherence length and spatial resolution. The inset in Figure 4 a shows a diffraction pattern for $10^{3}$ electrons per pulse averaged over $10^{6}$ pulses, i. e., $\sim 15 \mathrm{~min}$ at $1 \mathrm{kHz}$. The electron beam was focused on the detector, which resulted in a nearly collimated beam at the position of the sample. The typical diffraction rings of a polycrystalline sample were observed [1]. The corresponding radial distribution in dependence of momentum transfer $s$ is plotted in Figure 4 a. The peaks can be assigned to the allowed face-centered cubic crystal structure reflections for aluminum [1]. The corresponding Miller indices $(h k l)$ are used to label the peaks. a
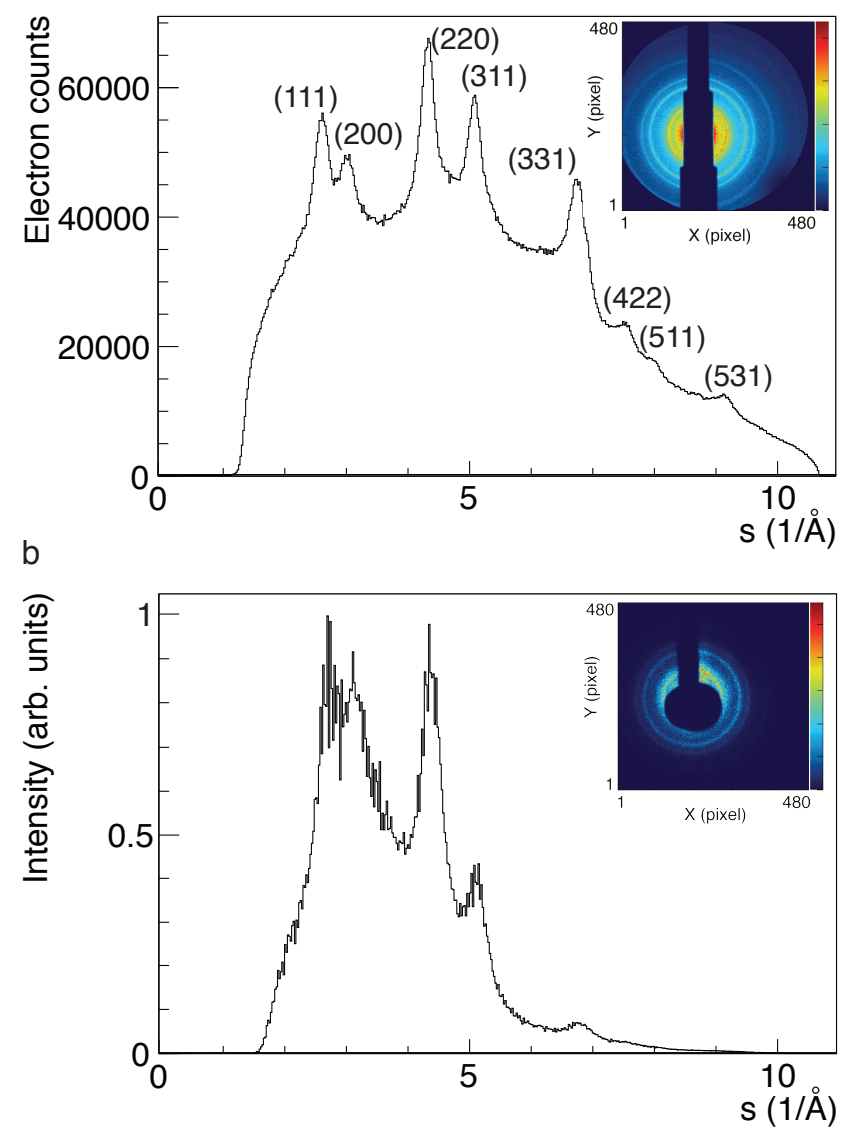

FIG. 4. Radial scattering intensity for aluminum for (a) $10^{3}$ and (b) $10^{6}$ electrons per pulse. Peaks are labeled with Miller indices $(h k l)$. The insets show the corresponding diffraction patterns.

The inset in Figure $4 \mathrm{~b}$ shows a diffraction pattern averaged over $10^{3}$ pulses $(\sim 1 \mathrm{~s})$ with $10^{6}$ electrons per pulse. A 6-mm-wide beam block was used, but the MCP voltage still had to be reduced to avoid damaging the detector due to many electrons scattered to small $s$. With this lower gain, single electrons could not be detected and the gain of the detector was not linear. Therefore, the peak intensities in Figure $4 \mathrm{a}$ and $\mathrm{b}$ cannot be compared quantitatively. Diffraction peaks are still visible in Figure $4 \mathrm{~b}$, except for the largest $s$ where the electron number and the gain were too small. This implies that the transverse coherence of the electron pulses were larger than $234 \mathrm{pm}$, where approximately $1 \mathrm{~nm}$ was expected from simulations. The spatial resolution of the imaging experiment was better than $234 \mathrm{pm}$, the interatomic distance corresponding to the (111) reflection in the diffraction pattern of aluminum. The restriction in resolution due to the lower detector gain will not occur in the envisioned gas-phase experiments, as the sample density will be much smaller. Thus, single-electron detection will also be possible for high electron numbers per pulse. 
Using the implemented spectrometer it was possible to experimentally obtain the emittance, i. e., the initial transverse spatial and velocity distributions of the electrons. The combination with simulations allowed for the deduction of further values, such as the coherence length and pulse duration of the propagated electron pulses. Compared to other sources with time resolutions on the order of 1 ps or below [7, 8], our setup did produce electron pulses with 1.5 ps duration for few electrons/pulse. More importantly, did table-top setup allow for a stable production of $>10^{6}$ electrons/pulse with estimated pulse durations of $60 \mathrm{ps}$ with a repetition rate of $1 \mathrm{kHz}$. Moreover, focusing, coherence, and the resolution in a diffraction experiments were confirmed to also be sufficient for the envisioned experiments to image dilute samples of controlled molecules [32].

The setup could be improved by increasing the acceleration fields: This would increase the electron number for the same laser power and lead to a smaller emittance and, thus, an increased coherence length of the electron beam. The time resolution could either be improved through a more compact design and stronger electric fields [13] or by combining the setup with an RF cavity for appropriate phase-space rotation for temporal focusing [12, 14, 41].

In conclusion, a new source for picosecond timeresolved electron diffraction experiments with the need for high numbers of electrons was described. It will allow, for instance, the investigation of dilute samples of controlled gas-phase molecules [32]. Moreover, enabled by its velocity map imaging spectrometer geometry, the setup allows to characterize electron beam properties, e. g., spatial and velocity distributions of the electrons. Coherence properties of the electron pulses were determined through simulations and through diffraction experiments of aluminum-foil samples to be sufficient for controlled molecule diffractive imaging.

We thank Sven Lederer, Ingo Hansen, and HansHinrich Sahling for helpful advise regarding the photocathode design. We gratefully acknowledge discussions with Martin Centurion, Jie Yang, Stephan Stern, and Henry N. Chapman on gas-phase aligned-molecule diffraction as well as helpful discussions regarding electron diffraction with Stuart Hayes, German Sciaini, Kostyantyn Pichugin, Julian Hirscht, and Dwayne Miller. We thank Masaki Hada for the preparation of the aluminum sample.

Besides DESY, this work has been supported by the excellence cluster "The Hamburg Center for Ultrafast Imaging - Structure, Dynamics and Control of Matter at the Atomic Scale" of the Deutsche Forschungsgemeinschaft (CUI, DFG-EXC1074) and the European Research Council through the Consolidator Grant 614507-COMOTION. N.L.M.M. gratefully acknowledges a fellowship of the Joachim Herz Stiftung. jochen.kuepper@cfel.de molecule-imaging.org

https://www.controlled-

1] L. Germer, Phys. Rev. 56, 58 (1939)

[2] K. Takayanagi, J. Vac. Sci. Technol. A 3, 1502 (1985)

[3] K. Lonsdale, Proc. Royal Soc. London A 123, 494 (1929)

[4] A. Yonath, Annu. Rev. Biophys. Biomol. Struct. 31, 257 (2002)

[5] I. Hargittai and M. Hargittai, Stereochemical Applications of Gas-Phase Electron Diffraction (VCH Verlagsgesellschaft, 1988).

[6] J. C. Williamson, J. M. Cao, H. Ihee, H. Frey, and A. H. Zewail, Nature 386, 159 (1997).

[7] H. Ihee, V. Lobastov, U. Gomez, B. Goodson, R. Srinivasan, C. Ruan, and A. H. Zewail, Science 291, 458 (2001)

[8] B. J. Siwick, J. R. Dwyer, R. E. Jordan, and R. J. D. Miller, Science 302, 1382 (2003)

[9] H. N. Chapman, A. Barty, M. J. Bogan, S. Boutet, S. Frank, S. P. Hau-Riege, S. Marchesini, B. W. Woods, S. Bajt, W. H. Benner, L. W. A., E. Plönjes, M. Kuhlmann, R. Treusch, S. Düsterer, T. Tschentscher, J. R. Schneider, E. Spiller, T. Möller, C. Bostedt, M. Hoener, D. A. Shapiro, K. O. Hodgson, D. van der Spoel, F. Burmeister, M. Bergh, C. Caleman, G. Huldt, M. M. Seibert, F. R. N. C. Maia, R. W. Lee, A. Szöke, N. Timneanu, and J. Hajdu, Nat. Phys. 2, 839 (2006)

[10] J. C. H. Spence, U. Weierstall, and H. N. Chapman, Rep. Prog. Phys. 75, 102601 (2012)

[11] A. Barty, J. Küpper, and H. N. Chapman, Annu. Rev. Phys. Chem. 64, 415 (2013)

[12] T. van Oudheusden, P. L. E. M. Pasmans, S. B. van der Geer, M. J. de Loos, M. J. van der Wiel, and O. J. Luiten, Phys. Rev. Lett. 105, 264801 (2010)

[13] M. S. Robinson, P. D. Lane, and D. A. Wann, Rev. Sci. Instrum. 86, 013109 (2015)

[14] G. Sciaini and R. J. D. Miller, Rep. Prog. Phys. 74, 096101 (2011)

[15] P. Musumeci, J. T. Moody, C. M. Scoby, M. S. Gutierrez, H. A. Bender, and N. S. Wilcox, Rev. Sci. Instrum. 81, 013306 (2010)

[16] S. Lahme, C. Kealhofer, F. Krausz, and P. Baum, Struct. Dyn. 1, 034303 (2014)

[17] M. W. van Mourik, W. J. Engelen, E. J. D. Vredenbregt, and O. J. Luiten, Struct. Dyn. 1, 034302 (2014)

[18] A. J. McCulloch, D. V. Sheludko, S. D. Saliba, S. C. Bell, M. Junker, K. A. Nugent, and R. E. Scholten, Nat. Phys. 7, $785(2011)$

[19] M. Gulde, S. Schweda, G. Storeck, M. Maiti, H. K. Yu, A. M. Wodtke, S. Schäfer, and C. Ropers, Science 345, 200 (2014).

[20] T. Zuo, A. D. Bandrauk, and P. B. Corkum, Chem. Phys. Lett. 259, 313 (1996).

[21] C. I. Blaga, J. Xu, A. D. DiChiara, E. Sistrunk, K. Zhang, P. Agostini, T. A. Miller, L. F. DiMauro, and C. D. Lin, Nature 483, 194 (2012).

[22] S. Ryu, R. Stratt, and P. Weber, J. Phys. Chem. A 107, 6622 (2003)

[23] J. C. H. Spence and R. B. Doak, Phys. Rev. Lett. 92, 198102 (2004)

[24] S. Pabst, P. J. Ho, and R. Santra, Phys. Rev. A 81, 043425 (2010)

[25] F. Filsinger, G. Meijer, H. Stapelfeldt, H. Chapman, and J. Küpper, Phys. Chem. Chem. Phys. 13, 2076 (2011) 
[26] J. Küpper, S. Stern, L. Holmegaard, F. Filsinger, A. Rouzée, A. Rudenko, P. Johnsson, A. V. Martin, M. Adolph, A. Aquila, S. Bajt, A. Barty, C. Bostedt, J. Bozek, C. Caleman, R. Coffee, N. Coppola, T. Delmas, S. Epp, B. Erk, L. Foucar, T. Gorkhover, L. Gumprecht, A. Hartmann, R. Hartmann, G. Hauser, P. Holl, A. Hömke, N. Kimmel, F. Krasniqi, K.U. Kühnel, J. Maurer, M. Messerschmidt, R. Moshammer, C. Reich, B. Rudek, R. Santra, I. Schlichting, C. Schmidt, S. Schorb, J. Schulz, H. Soltau, J. C. H. Spence, D. Starodub, L. Strüder, J. Thøgersen, M. J. J. Vrakking, G. Weidenspointner, T. A. White, C. Wunderer, G. Meijer, J. Ullrich, H. Stapelfeldt, D. Rolles, and H. N. Chapman, Phys. Rev. Lett. 112, 083002 (2014) arXiv:1307.4577 [physics]

[27] S. Stern, L. Holmegaard, F. Filsinger, A. Rouzee, A. Rudenko, P. Johnsson, A. V. Martin, A. Barty, C. Bostedt, J. Bozek, R. Coffee, S. Epp, B. Erk, L. Foucar, R. Hartmann, N. Kimmel, K.-U. Kühnel, J. Maurer, M. Messerschmidt, B. Rudek, D. Starodub, J. Thøgersen, G. Weidenspointner, T. A. White, H. Stapelfeldt, D. Rolles, H. N. Chapman, and J. Küpper, Faraday Disc. 171, 393 (2014) arXiv:1403.2553 [physics]

[28] C. J. Hensley, J. Yang, and M. Centurion, Phys. Rev. Lett. 109, $133202(2012)$

[29] J. Yang, V. Makhija, V. Kumarappan, and M. Centurion, Struct. Dyn. 1, 044101 (2014)

[30] L. Holmegaard, J. H. Nielsen, I. Nevo, H. Stapelfeldt, F. Filsinger, J. Küpper, and G. Meijer, Phys. Rev. Lett. 102, 023001 (2009) arXiv:0810.2307 [physics.chem-ph]

[31] S. Trippel, T. Mullins, N. L. M. Müller, J. S. Kienitz, J. J. Omiste, H. Stapelfeldt, R. González-Férez, and J. Küpper, Phys. Rev. A 89, 051401(R) (2014) arXiv:1401.6897 [quant- ph]

[32] Y.-P. Chang, D. Horke, S. Trippel, and J. Küpper, Int. Rev. Phys. Chem. (2015), submitted, arXiv:1505.05632 [physics]

[33] T. Kierspel, J. Wiese, T. Mullins, J. Robinson, A. Aquila, A. Barty, R. Bean, R. Boll, S. Boutet, P. Bucksbaum, H. N. Chapman, L. Christensen, A. Fry, M. Hunter, J. E. Koglin, M. Liang, V. Mariani, A. Morgan, A. Natan, V. Petrovic, D. Rolles, A. Rudenko, K. Schnorr, H. Stapelfeldt, S. Stern, J. Thøgersen, C. H. Yoon, F. Wang, S. Trippel, and J. Küpper, J. Phys. B (2015), submitted for special issue on "Science at Free-Electron Lasers".

[34] H. Stapelfeldt and T. Seideman, Rev. Mod. Phys. 75, 543 (2003)

[35] S. Trippel, T. Mullins, N. L. M. Müller, J. S. Kienitz, K. Długołęcki, and J. Küpper, Mol. Phys. 111, 1738 (2013). arXiv:1301.1826 [physics.atom-ph]

[36] M. Stei, J. von Vangerow, R. Otto, A. H. Kelkar, E. Carrascosa, T. Best, and R. Wester, J. Chem. Phys. 138, 214201 (2013).

[37] A. R. Bainbridge and W. A. Bryan, New J. Phys. 16, 103031 (2014)

[38] J. R. Maldonado, P. Pianetta, D. H. Dowell, J. Corbett, S. Park, J. Schmerge, A. Trautwein, and W. Clay, Astrophys. Lett. \& Comm. 101, 231103 (2012)

[39] K. Floettmann, "ASTRA, a space charge tracking algorithm,' (1997).

[40] S. Flügge, Rechenmethoden der Elektrodynamik. Aufgaben mit Lösungen (Springer Verlag, 1986).

[41] F. Fu, S. Liu, P. Zhu, D. Xiang, J. Zhang, and J. Cao, Rev. Sci. Instrum. 85, 083701 (2014) 